\title{
Algoritmo de reconstrução de imagens para um sistema de Tomografia por Impedância Elétrica (TIE) baseado em configuração multiterminais
}

\author{
Felipe Dalvi-Garcia*, Marcio Nogueira de Souza, Alexandre Visintainer Pino
}

Resumo Introdução: A Tomografia por Impedância Elétrica (TIE) é um método de imageamento de baixo custo, não invasivo, portátil e livre de radiação ionizante, que é capaz de mapear a distribuição das propriedades elétricas de tecidos biológicos. Apesar da baixa resolução das imagens reconstruídas, quando comparadas a outras técnicas de imageamento tomográfico, tem-se observado uma série de aplicações clínicas nas quais a EIT é utilizada para monitorar a atividade de determinados tecidos do corpo humano. A maioria dos sistemas de EIT comerciais tem sido pautada no paradigma originalmente proposto de uma configuração multiportas. Este trabalho apresenta um algoritmo de reconstrução de imagem para um sistema alternativo de EIT que usa uma configuração multiterminais. Métodos: $\mathrm{O}$ algoritmo é baseado na associação entre o plano tomográfico do volume condutor e um sistema eletrostático análogo. Para avaliar o algoritmo, imagens de EIT foram reconstruídas a partir de dados obtidos experimentalmente em tanque com solução salina, sendo as mesmas quantificadas por meio de métricas estabelecidas para este propósito. Resultados: Quantitativamente, as imagens reconstruídas com o algoritmo proposto apresentaram um erro de posição de $\pm 10 \%$, resolução e deformação decrescentes do centro para a borda e oscilação em torno de $60 \%$ do meio. Conclusão: Uma vez que o algoritmo não possui nenhum processo de filtragem, os resultados foram considerados satisfatórios e encorajadores para trabalhos futuros visando melhorias.

Palavras-chave Tomografia por Impedância Elétrica, Bioimpedância, Sistema multiterminais.

\section{Image reconstruction algorithm for a multiterminal-based Electrical Impedance Tomography (EIT) system}

\begin{abstract}
Introduction: Electrical Impedance Tomography (EIT) is a low cost, non-invasive, portable and free of ionizing radiation method for imaging the distribution of the electric properties of biological tissues. Despite the low resolution of the reconstructed images when compared with other tomographic techniques, there is a range of clinical applications in which the EIT has been used to monitor the activity of certain body tissues. Most of commercial EIT systems are based on the original paradigm defined as a multiport configuration. This paper presents an algorithm developed to reconstruct images for an alternative multiterminal-based EIT system. Methods: The algorithm is based on the association between the tomographic plane of the conductor volume and an analogous electrostatic system. To evaluate the algorithm, EIT images were reconstructed using data obtained experimentally in a saline solution tank, which were quantified by some metrics established for this purpose. Results: Quantitatively, EIT images reconstructed by the proposed algorithm presented a position error around $\pm 10 \%$, resolution and shape deformation decreasing from center to the edge, and ringing around $60 \%$ of the medium. Conclusion: Taking into account that no filtering was used in the algorithm, the results can be considered satisfactory and encourage future works aiming further improvement.
\end{abstract}

Keywords Electrical Impedance Tomography, Bioimpedance, Multiterminal-based system. 


\section{Introdução}

A Tomografia por Impedância Elétrica (EIT - do inglês Electrical Impedance Tomography) é uma técnica não invasiva de imageamento da estrutura interna de volumes condutores baseada na distribuição de condutividade (ou de resistividade), que por sua vez é estimada a partir das respostas do volume condutor à aplicação de excitação de tensão ou de corrente em eletrodos posicionados ao seu redor. Em geral, a injeção do sinal de excitação ocorre em um determinado eletrodo (ou par de eletrodos) na superfície do volume condutor, enquanto a resposta correspondente é coletada pelos demais eletrodos (ou pares de eletrodos). Esse processo se repete para vários conjuntos de excitação e de resposta, de acordo com um protocolo de aquisição predefinido, até que se colete o maior número possível de informações a respeito da distribuição de condutividade (ou resistividade). Essas informações são, então, processadas por um algoritmo de reconstrução, dando origem a uma imagem de um corte tomográfico baseada na distribuição de condutividade da secção transversal do volume condutor que passa pelo plano dos eletrodos circundantes. Uma variante da técnica pode também ser usada para a geração de imagens tomográficas tridimensionais.

Algumas das primeiras imagens de EIT de humanos publicadas foram obtidas com o protótipo desenvolvido por Brown et al. (1985), do Departamento de Física Médica e Engenharia Clínica da Universidade de Sheffield (Reino Unido). Neste trabalho, os autores usaram 16 eletrodos, organizados em pares adjacentes, para a injeção de uma corrente de excitação e medição das respostas de tensão nos pares restantes. As medidas realizadas foram posteriormente processadas por um algoritmo baseado no método de retroprojeção para reconstruir as imagens de EIT. Embora as imagens tivessem baixa resolução, as possíveis aplicações em pesquisa e na área clínica começaram a ser consideradas Brown et al. (1985). Desde então, um intenso esforço tem sido empregado no sentido de aprimorar essa técnica de imageamento, tanto sob o aspecto da instrumentação de excitação e aquisição envolvida, quanto dos algoritmos para reconstrução das imagens.

Apesar de potencialmente atrativa, as principais limitações da EIT continuam sendo a baixa resolução das imagens, a qual está relacionada com o número de eletrodos, e o custo de tempo para a reconstrução do tomograma, seja por restrições de hardware ou pelo custo computacional do algoritmo utilizado (Holder, 2004). Não obstante, a EIT pode ser vista como um método alternativo a outras técnicas de imageamento interno, tais como Raios-X, Ressonância Magnética, Tomografia Computadorizada e Ultra-Som, no sentido de ser de baixo custo, não ser invasiva, não submeter o paciente a nenhum tipo de radiação ionizante e por apresentar grande portabilidade, além de permitir a monitoração do paciente por um longo período de tempo.

Vários grupos de pesquisa têm desenvolvido sistemas de EIT para uma ampla diversidade de aplicações em saúde. Dentre os mais recentes trabalhos, podem ser citados os do grupo de College London e de Sheffield - UK, para monitoração da atividade pulmonar e cerebral (Bagshaw et al., 2003; Liston, 2003; Nebuya et al., 2011; Wilson et al., 2001; Yerworth et al., 2003); os de Rensselaer - EUA, com aplicações em mamografia, monitoração de perfusão pulmonar e atividade cardíaca (Ardrey et al., 2011; Choi et al., 2008; Liu, 2007; Saulner et al., 2007); os de Dartmouth - EUA, para detecção de câncer de mama e imageamento de próstata (Borsic et al., 2010; Halter et al., 2008) e os da Universidade de Kyung Hee - KO (Oh et al., 2007, 2008).

Grande parte dos tomógrafos de EIT existentes se baseiam no modelo de aquisição de Sheffield, denominada configuração multiportas. Entretanto, observa-se que nesse tipo de configuração as linhas de campo elétrico se tornam escassas e dispersas na região central do planeo tomográfico; sendo que outras configurações de eletrodos têm sido propostas para minimizar tal problema, tais como, por exemplo, a configuração multiterminais (Kauati et al. 1998; Kauati e Souza, 1999).

Quanto aos algoritmos de reconstrução, uma possível classificação para os diversos métodos propostos consiste em caracterizá-los como sendo iterativos ou não-iterativos. Os algoritmos iterativos em geral utilizam modelos com elementos finitos e técnicas de minimização de erros, tais como o de Gauss-Newton (Hua et al., 1991; Linderholm et al., 2008; Ortega, 1970), para alcançar resultados mais precisos em detrimento do custo computacional, que está diretamente relacionado ao tempo de convergência (Holder, 2004). Já dentre os métodos não-iterativos os de maior destaque são os chamados algoritmos de um passo, tendo como principais exemplos o algoritmo de retroprojeção (Barber et al., 1984; Brown et al., 1985) e o de Newton de um passo (Cheney et al., 1990; Isaacson et al., 1992), implementados essencialmente nos sistemas de Sheffield e Rensselaer, respectivamente. Nessa abordagem, o problema da reconstrução é linearizado, permitindo a geração da tomografia em um tempo relativamente menor que os algoritmos iterativos. Como consequência dessas simplificações, as imagens resultantes perdem em qualidade e em exatidão. Porém, em certas aplicações clínicas, tais como monitoração da atividade cardíaca 
e da ventilação pulmonar, em que o tempo é um fator limitante, o uso de algoritmos de um passo é mais adequado.

O presente trabalho propõe um algoritmo de reconstrução de imagens de EIT de um passo baseado em conceitos de analogia eletrostática e que foi desenvolvido para ser aplicado em dados fornecidos por um sistema de EIT alternativo baseado na configuração multiterminais (Kauati et al. 1998; Kauati e Souza, 1999). Além dos princípios que norteiam o algoritmo, são apresentados resultados de imagens de ETI obtidas a partir de dados relacionados a experimentos em tanques de solução salina. Ao final, são discutidas as vantagens e desvantagens dessa abordagem com base na avaliação de figuras de mérito para imagens de EIT.

\section{Métodos}

\section{Configuração multiportas e multiterminais}

$\mathrm{Na}$ configuração multiportas, uma corrente de excitação é aplicada a um par de eletrodos adjacentes e as diferenças de potencial resultantes são medidas nos demais pares de eletrodos. Já na abordagem multiterminais, a corrente de excitação é injetada em um eletrodo (terminal) e as correntes de transferência são medidas nos demais eletrodos que se encontram aterrados (Kauati e Souza, 1999). Na Figura 1 estão representadas ambas as configurações, bem como um esboço do comportamento das linhas de campo elétrico e equipotenciais no plano tomográfico.
Um sistema multiterminais é normalmente definido por uma matriz de admitâncias, já que para este caso os valores medidos são as correntes nos terminais e a tensão na fonte de corrente. Assim,

$\left[\begin{array}{c}i_{1} \\ i_{2} \\ \vdots \\ i_{L}\end{array}\right]=\left[\begin{array}{cccc}y_{11} & y_{12} & \ldots & y_{1 L} \\ y_{21} & y_{22} & \ldots & y_{2 L} \\ \vdots & \vdots & \ddots & \vdots \\ y_{L 1} & y_{L 2} & \ldots & y_{L L}\end{array}\right] \cdot\left[\begin{array}{c}v_{1} \\ v_{2} \\ \vdots \\ v_{L}\end{array}\right]$

ou, matricialmente, $\mathbf{I}=\mathbf{Y}_{c} \cdot \mathbf{V}$

onde I é o vetor com as correntes que fluem pelos terminais $(L \times 1), \mathbf{V}$ é o vetor com as tensões em cada terminal $(L \times 1)$, e $\mathbf{Y}_{c}$ é a matriz com as admitâncias de curto-circuito $(L \times L)$, em que cada elemento $y_{i j}$ é definido como:

$y_{i j}=\frac{i_{j}}{v_{i}}, \operatorname{com} v_{k}=0, k=1,2, \ldots, L$ e $k \neq i$

Note-se que se os elementos da $i$-ésima linha de $\mathbf{Y}_{c}$ foram divididos por $y_{i i}$ (ou seja, o elemento da diagonal), obtém-se a fração da corrente total que flui pelos eletrodos aterrados.

\section{Método para estimação da condutividade relativa}

A ideia do algoritmo desenvolvido é obter um modelo representativo da secção transversal do volume condutor baseado em conceitos de analogia eletrostática. Partindo desse princípio, suponha-se um plano homogêneo isotrópico $\prod_{0} \in \mathfrak{R}^{2}$, circular, com condutividade $\sigma_{0}$. Se um plano for puramente
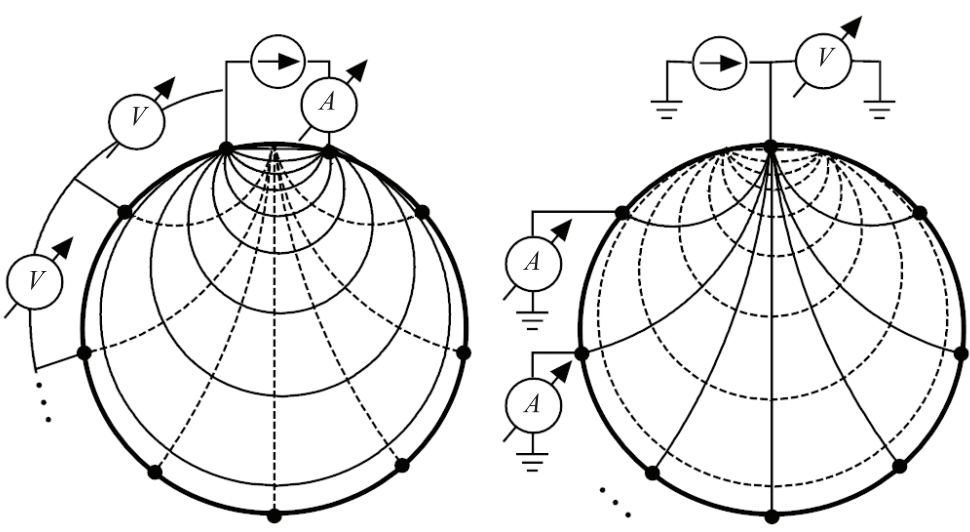

Figura 1. Plano resistivo em uma configuração multiportas (à esquerda) e multiterminais (à direita). As equifluxos ou linhas de campo estão representadas por linhas cheias, enquanto que as equipotenciais por linhas tracejadas. No caso da configuração multiportas, a corrente é injetada em um par de eletrodos adjacentes, enquanto que as diferenças de potencial são medidas nos demais pares. Já na configuração multiterminais, a tensão é medida sobre a fonte que injeta corrente em apenas um eletrodo, enquanto são medidas as correntes que fluem para os demais eletrodos que estão aterrados.

Figure 1. Resistive plane in multi-port (left) and multiterminal (right) configuration. The equiflux lines are the solid lines and the equipotential lines are the dashed lines. In multi-port configuration, current is injected into a pair of adjacent electrodes, while the potential differences are measured in other pairs. In contrast, in multiterminal configuration, the voltage is measured on the source that injects current in only one electrode while the currents that flow to other grounded electrodes are measured. 
resistivo, livre de fontes internas dependentes ou independentes e a corrente injetada for de baixa frequência $(<100 \mathrm{kHz})$, a relação entre o campo potencial elétrico $\Phi$ e a distribuição de condutividade é expressa pela Equação de Poisson (Eyüboglu, 2006):

$\nabla(\sigma \nabla \Phi)=0$

com condição de contorno

$J_{n}=\sigma \frac{\partial \phi}{\partial n}=0$, na borda de $\prod_{0}$

em que $J_{n}$ é a componente normal da densidade de corrente e $\partial \Phi / \partial n$ é a derivada da função potencial na direção normal a uma linha de $\Phi$ constante. Quando a condutividade é constante ao longo do plano, a Equação 3 se reduz à Equação de Laplace:

$\nabla^{2} \Phi=0$

Note-se que a Equação acima pode ser reescrita como:

$\nabla \cdot \mathbf{E}=0$

onde $\mathbf{E}$ é o campo elétrico em $\Pi_{0}$. O divergente representado na Equação 6 indica que, segundo a Lei de Gauss, o fluxo elétrico total $\psi$ através de uma superfície fechada qualquer que contenha $\prod_{0}$ é nulo. Adicionalmente, deve-se considerar ainda que não há fluxo de corrente para fora de $\Pi_{0}$. Logo,

$J_{n}=0$

em todos os pontos da borda de $\prod_{0}$, com exceção dos pontos referentes às posições dos eletrodos, através dos quais há passagem de corrente.

Uma aproximação eletrostática que atende às restrições impostas pelas Equações 6 e 7, é aquela associada a um problema eletrostático em um meio com permissividade $\varepsilon_{0}$ (vácuo) e onde os valores de corrente injetados e coletados ao redor de $\Pi_{0}$ são atribuídos a $L$ linhas de densidade de carga $\lambda_{0 l}$ posicionadas nas coordenadas dos eletrodos, e suas cargas imagem $\lambda_{0 l}$ são localizadas na posição conjugada simétrica em relação à borda, sendo todas de comprimento $h \rightarrow \infty$ (Figura 2). Uma vez que $\lambda_{0 l} \mathrm{e}$ $\lambda_{0 l}$ possuam os mesmos módulos e sinais, pelo método das imagens (Bertemes-Filho, 2006; Durand, 1964) conclui-se que pelo ponto médio da distância entre elas, ou seja, num círculo com a geometria da borda do plano $\prod_{0}$, não haverá campo elétrico normal, ou seja, pelo círculo que define a borda de $\Pi_{0}$ passa uma equifluxo. Isso significa dizer que as linhas de campo permanecem confinadas dentro de um domínio que respeita a geometria de $\Pi_{0}$, o que do ponto de vista eletrodinâmico significa dizer que não há fluxo

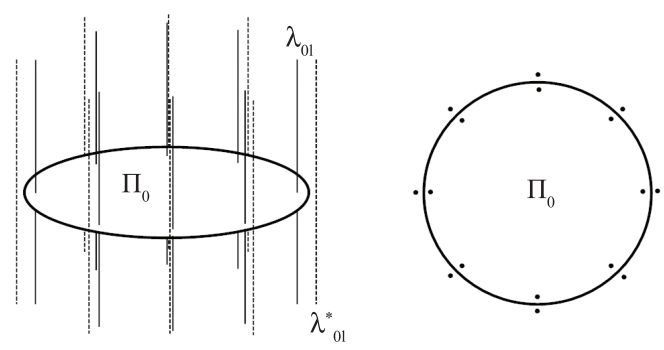

Figura 2. Vista em perspectiva (à esquerda) e vista superior (à direita) da distribuição de $L$ linhas de densidade de carga igualmente espaçadas ao redor de um contorno semelhante aquele associado ao plano $\prod_{0}$. As cargas imagens aparecem tracejadas.

Figure 2. Perspective (left) and superior (right) views of the distribution of $L$ linear density charges equally displaced around a boundary similar to the $\prod_{0}$ plane. The image charges are the dashed lines.

de corrente para fora de $\prod_{0}$. Além disso, como as linhas equipotenciais são perpendiculares às linhas equifluxos, a derivada direcional $\partial \Phi / \partial n$ em qualquer ponto da borda de $\prod_{0}$ é nula, atendendo à condição dada na Equação 7.

$\mathrm{O}$ valor do vetor campo elétrico $\mathbf{E}$ em cada ponto de coordenadas complexas $z=x+j y$ em $\prod_{0}$ pode ser calculado pela Equação 8 (Durand, 1964):

$$
\begin{aligned}
& \mathrm{E}=\frac{1}{2 \pi \varepsilon_{0}} \sum_{l=1}^{L}\left[\lambda_{0 l}\left(\frac{z-z e_{l}}{\left|z-z e_{l}\right|^{2}}\right)+\lambda_{0 l}^{*}\left(\frac{z-z e_{l}^{*}}{\left|z-z e_{l}^{*}\right|^{2}}\right)\right] \\
& z \neq z e_{l} \mathrm{e} z \neq z e_{l}^{*}
\end{aligned}
$$

onde $\varepsilon_{0}$ é a permissividade no vácuo e $z e_{l}$ e $z e_{l}^{*}$ são, respectivamente, as coordenadas complexas (para um sistema com origem no centro do círculo que descreve o plano $\Pi_{0}$ ) da $l$-ésima linha de carga e de sua imagem. Como os valores das cargas no problema eletrostático correspondem às parcelas de corrente que fluem pelos $(L-1)$ eletrodos aterrados quando uma fonte de corrente de amplitude suposta unitária é aplicada em um dos $L$ eletrodos, a Lei de Kichhoff estabelece que

$\lambda_{0 i}+\sum_{\substack{l=1, l \neq i}}^{N} \lambda_{0 l}=0$

Observe-se que os valores das cargas são linearmente dependentes, em decorrência do fato de que se houver um decréscimo de corrente em um dos eletrodos em relação a um estado inicial, essa diferença será distribuída entre as parcelas de corrente que fluem pelos outros eletrodos, de modo que a igualdade da Equação 9 se mantenha. Além disso, já que as linhas têm comprimento $h \rightarrow \infty$, o vetor campo elétrico resultante na Equação 8 possui apenas componentes em $\mathfrak{R}^{2}$. No problema eletrostático semelhante do plano resistivo $\prod_{0}$ a distribuição de cargas é tal que o formato das linhas de campo se 
aproxima do apresentado na Figura 4. Ademais, sabe-se que o vetor campo elétrico calculado pela Equação 8 para cada ponto $z$ é tangente à linha de campo (equifluxo) que passa por $z$, e que tal linha "sai de" ou "entra em" uma das linhas de densidade de carga, em função do arranjo geométrico dessas cargas e de seu valor. Pode-se dizer então que cada equifluxo $\psi_{i, z}^{l}$ (equifluxo que sai da carga $i$, passa pelo ponto $z$ e vai para a linha de densidade de carga l) contribui como uma parcela do fluxo elétrico total que vai para a $l$-ésima linha de densidade de carga. É possível obter experimentalmente, ou analiticamente segundo alguma aproximação, os valores de carga para o caso de um plano homogêneo e com isso, a partir da Equação 8, traçar iterativamente as equifluxos de um ponto a outro, conforme mostrado na Figura 3.

Seja uma superfície gaussiana cilíndrica colocada ao redor de uma das linhas de densidade de carga $\lambda_{0}$ no problema eletrostático semelhante do plano $\prod_{0}$. Pela Lei de Gauss, o fluxo elétrico total nessa carga é (para simplificar a notação, a carga imagem $\lambda_{0 l}$ será omitida, uma vez que ela contribui para os cálculos de igual maneira a $\lambda_{0 l}$ ):

$\Psi_{0 l}=\frac{\lambda_{0 l} h}{\varepsilon_{0}}$

Como exemplo, observe-se na Figura 4 a superfície cilíndrica ao redor da linha de densidade de carga $l$, quando a densidade $\lambda_{0 i}=1$, ou seja, quando no plano tomográfico condutivo a corrente é injetada no $i$-ésimo eletrodo e a fração nos demais é obtida a partir de $\mathbf{Y}$, como descrito na seção anterior. Note-se que há uma quantidade finita de equifluxos que vai de $\lambda_{0 i}$ a $\lambda_{0 l}$, que tem sinal negativo e módulo menor que 1, para satisfazer à Equação 9. Pode-se dizer que o conjunto de equifluxos $\psi_{i, z}^{l}(l \neq i)$ formam um subconjunto $\varpi_{i, l}$ (Figura 5) no problema eletrostático semelhante ao plano $\prod_{0}$, na qual todos os pontos $z$ $\in \varpi_{i, l}$ estão sobre alguma das equifluxos $\psi_{i, z}^{l}$. Sendo assim, a parcela $\psi_{i z}^{l}$ do fluxo total $\psi_{0 /}$ que passa pelo ponto $z \in \varpi_{i, l}$ é dada por:

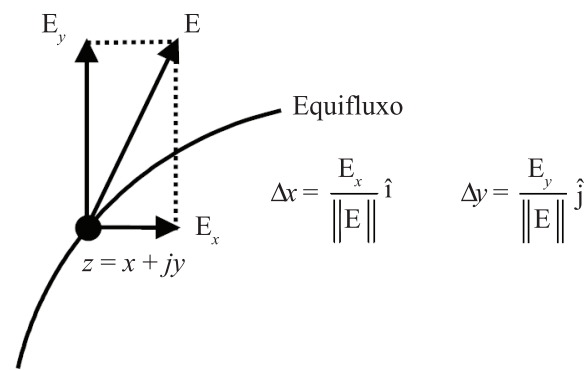

Figura 3. Campo elétrico em um ponto $z$ qualquer. A equifluxo que passa por $z$ pode ser traçada iterativamente tendo-se como passo os incrementos $\Delta x$ e $\Delta y$.

Figure 3. Electric field at a $z$ point. The equiflux through $z$ can be drawn iteratively with $\Delta x$ and $\Delta y$ step. $\psi_{i z}^{l}=k_{z} \psi_{0 l}$

$\psi_{i, z}^{l}=k_{z} \frac{\lambda_{0 l} h}{\varepsilon_{0}}$

onde $k_{z}$ é uma constante que representa a fração de $\psi_{0}$ correspondente a $\psi_{i, z}^{l}$ e que depende da posição de $z$.

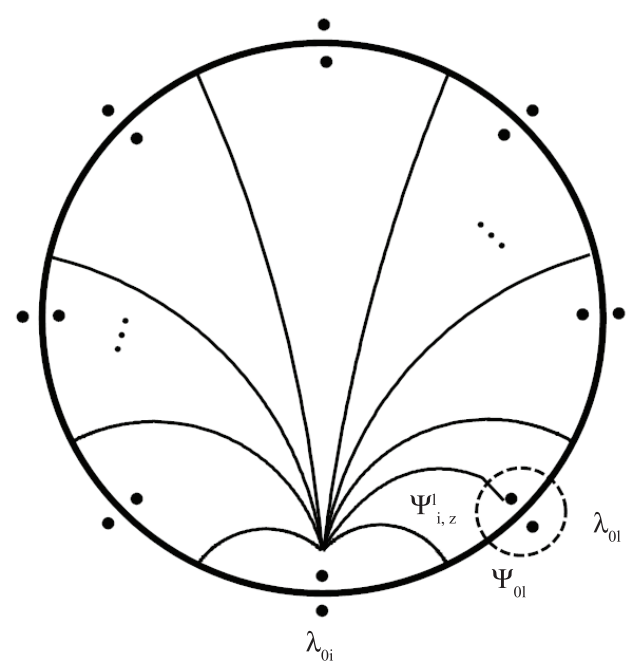

Figura 4. Superfície gaussiana cilíndrica (circunferência tracejada, vista de cima) colocada ao redor da linha de densidade de carga $\lambda_{0}$ para a qual existe um número finito de equifluxos $\psi_{i, z}^{l}$ que contribuem para o fluxo total $\psi_{0 /}$ em $\lambda_{0 /}$

Figure 4. Cylindrical gaussian surface (the dashed circumference) placed around the linear density charge $\lambda_{01}$ for which there is a finite number of equiflux $\psi_{i, z}^{l}$ that contributes to the total flux $\psi_{0 l}$ in $\lambda_{0 r}$

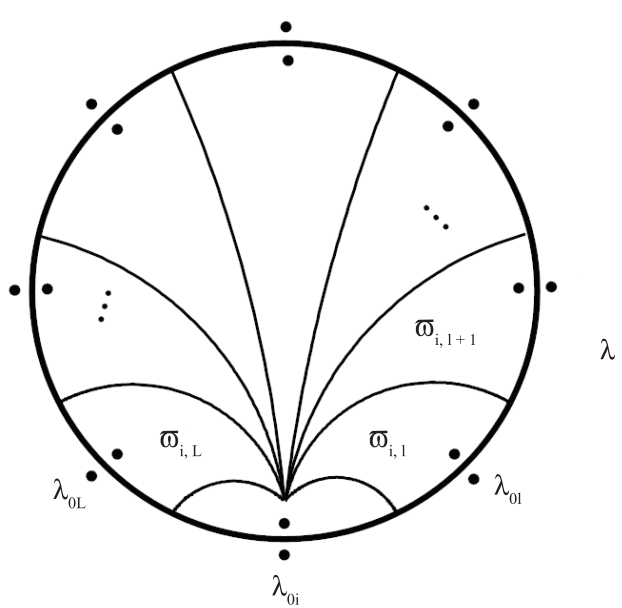

Figura 5. Subregiões $\varpi_{i,}$ no problema eletrostático semelhante ao plano $\prod_{0}$ que são formadas pelas equifluxos que vão da $i$-ésima até a $l$-ésima linha de densidade de carga. Essas equifluxos são traçadas iterativamente uma única vez para o caso de plano homogêneo. Figure 5. Subregions $\varpi_{.}$in the electrostatic problem similar to the $\Pi_{0}$ plane. They are formed by the equiflux that flow from the $i$-th to the l-th linear density charge. These equiflux are drawn iteratively once for the homogeneous plane. 
Suponha-se agora um novo plano condutor, denominado agora П (não mais homogêneo), no qual ocorreu uma variação de condutividade em relação ao plano homogêneo, ou seja, $\sigma=\sigma_{0}+\Delta \sigma$. Isso equivale, no problema eletrostático, a uma variação na permissividade $\varepsilon=\varepsilon_{0}+\Delta \varepsilon$. Como resultado dessa variação, ocorrerá um desbalanceamento das linhas de densidade de cargas, mantendo-se, no entanto, a dependência linear entre elas em decorrência da Equação 9. A Equação 8, porém, não é válida para meios não-homogêneos, de maneira que não é possível saber a forma das linhas de campo em função dessa perturbação. Contudo, pode-se supor que se $\Delta \varepsilon$ for pequena o suficiente, as sub-regiões $\varpi_{i, 1}$ terão aproximadamente a mesma forma que em $\prod_{0}$. Para que isso seja verdade, o fluxo elétrico total ao redor de cada linha de densidade de carga deve permanecer o mesmo. Já que a posição geométrica dessas linhas não se altera, o desbalanceamento em seus valores só pode ser explicado por uma variação correspondente na permissividade do subconjunto $\varpi_{i, l}$ Assim, para cada nova carga $\lambda_{l}=\lambda_{0 l}+\Delta \lambda_{l}$ deve haver um novo valor de permissividade $\varepsilon_{l}=\varepsilon_{0}+\Delta \varepsilon_{l}$ que mantenha constante a relação da Equação 10.

Ora, se o fluxo elétrico total da Equação 10 é suposto constante em cada linha de densidade de carga no meio $\Pi$, então as equifluxos $\psi_{i, z}^{l}$ têm os mesmos valores que no caso homogêneo. Logo, para um ponto $z$ qualquer, pode-se supor que:

$k_{z} \frac{\lambda_{l} h}{\varepsilon_{l}}=k_{z} \frac{\lambda_{0 l} h}{\varepsilon_{0}}$

Como a constante $k_{z}$ é a mesma para o ponto $z$ em ambos os casos e as cargas tem o mesmo comprimento $h$, a Equação 12 se restringe a:

$\frac{\varepsilon_{l}}{\varepsilon_{0}}=\frac{\lambda_{l}}{\lambda_{0 l}}, \forall z \in \varpi_{i, l}$

Considerando que para frequências da corrente de excitação da ordem de $10^{3} \mathrm{~Hz}$ ou menores (Gabriel et al., 1996) a condutividade de um meio condutor é diretamente proporcional à permissividade de um problema eletrostático análogo, a Equação 13 pode ser vista como a relação entre a condutividade $\sigma_{1}$ e a condutividade $\sigma_{0}$ do ponto $z \in \Phi_{i, l}$ Logo,

$\frac{\sigma_{l}}{\sigma_{0}}=\frac{\lambda_{l}}{\lambda_{0 l}}, \forall z \in \varpi_{i, l}$

Então, pelo princípio da superposição, pode-se obter a variação média de condutividade no plano perturbado $\prod$ somando-se as parcelas de variação em cada uma das $L$ aplicações de corrente em um ciclo completo. A Equação 14 é utilizada para determinar a relação de condutividade entre os casos perturbado e homogêneo teórico em cada ponto.

\section{Plataforma de testes e hardware utilizados}

Os experimentos de avaliação do algoritmo proposto foram realizados num tanque circular de solução salina de $29 \mathrm{~cm}$ de diâmetro interno com 16 eletrodos no formato de calotas esféricas de $7 \mathrm{~cm}$ de diâmetro, feitos de aço inoxidável e posicionados na borda, igualmente espaçados entre si. Para perturbação do meio homogêneo foram utilizados fantomas cilíndricos de PVC de 3,5 cm de diâmetro.

O sistema de EIT em configuração multiterminais utilizado para obtenção dos dados foi desenvolvido em nosso laboratório e é formado basicamente de cinco módulos: fonte de corrente, pré-amplificadores, sistema de chaveamento (controlador e circuito de chaveamento) e conversor A/D (Figura 6). A fonte da corrente de excitação foi senoidal de $50 \mathrm{kHz}$ e $1 \mathrm{~mA}_{\mathrm{pp}}$, obtida por meio de um oscilador ponte de Wien e um conversor tensão-corrente baseado no OTA CA3080 (Intersil, USA). O circuito de chaveamento era composto por multiplexadores analógicos que utilizaram relés mecânicos para garantir baixa resistência de contato. Como o objetivo deste trabalho foi a reconstrução de imagens e não otimização do tempo de aquisição dos dados, foi utilizada uma baixa frequência de chaveamento $(10 \mathrm{~Hz})$. Para coletar a corrente que flui por cada eletrodo, assim como para aterrá-los virtualmente, foram usados pré-amplificadores de transimpedância de valor de $2,6 \mathrm{k} \Omega$, construídos com amplificadores operacionais LF356 (Texas Instruments, USA). A conversão analógico-digital da tensão sobre a fonte de corrente de excitação, assim como dos sinais de saída dos pré-amplificadores de transimpedância, que foi realizada por uma placa PCI-6251 (16 bits de resolução, $1 \mathrm{MHz}$ de amostragem, National Instruments ${ }^{\circledR}$ ). O software para controle do chaveamento entre eletrodos, bem como para armazenamento dos dados coletados foi implementado em LabVIEW, também da National Instruments ${ }^{\mathbb{B}}$.

\section{Avaliação das imagens reconstruídas}

Os parâmetros usados para avaliação das imagens de EIT deste trabalho se baseiam em algumas das figuras de mérito propostas por Adler et al. (2009), as quais são brevemente descritas, por ordem de importância. Para tal, é importante considerar as grandezas e seus respectivos significados representados na Figura 7. A avaliação é feita sobre o conjunto de pixels $\mathbf{Z}_{0}$ formado a partir dos pontos da largura à meia altura (FWHM - Full Width at Half Maximum) da estimativa da distribuição de condutividade.

Erro de Posição (PE - Position Error): Mede o quanto a posição reconstruída da perturbação 
de condutividade, doravante chamada de objeto reconstruído, se aproxima da posição real do fantoma no tanque, tendo como referência os centros de gravidade de cada um. Sendo assim, define-se o erro de posição como:

$$
P E=r_{f}-r_{o}
$$

Quanto mais $P E$ varia ao longo do meio, menos confiável é a interpretação referente ao objeto reconstruído.
Oscilação (RNG - Ringing): Mede a parcela da imagem reconstruída fora do círculo $C$ cuja amplitude tem sinal oposto à amplitude da imagem dentro de $C$. $R N G=\frac{\sum_{k \notin C,[\mathbf{Z}]_{k}>0}\left[\mathbf{Z}_{o}\right]_{k}}{\sum_{k \in C}\left[\mathbf{Z}_{o}\right]_{k}}$

Note-se que o termo $[\mathbf{Z}]_{k}>0$ é usado para o caso em que a variação de condutividade do objeto reconstruído é negativa. Quando o fantoma for mais condutivo que o meio onde ele é inserido e, portanto, a condutividade do objeto for positiva em relação ao

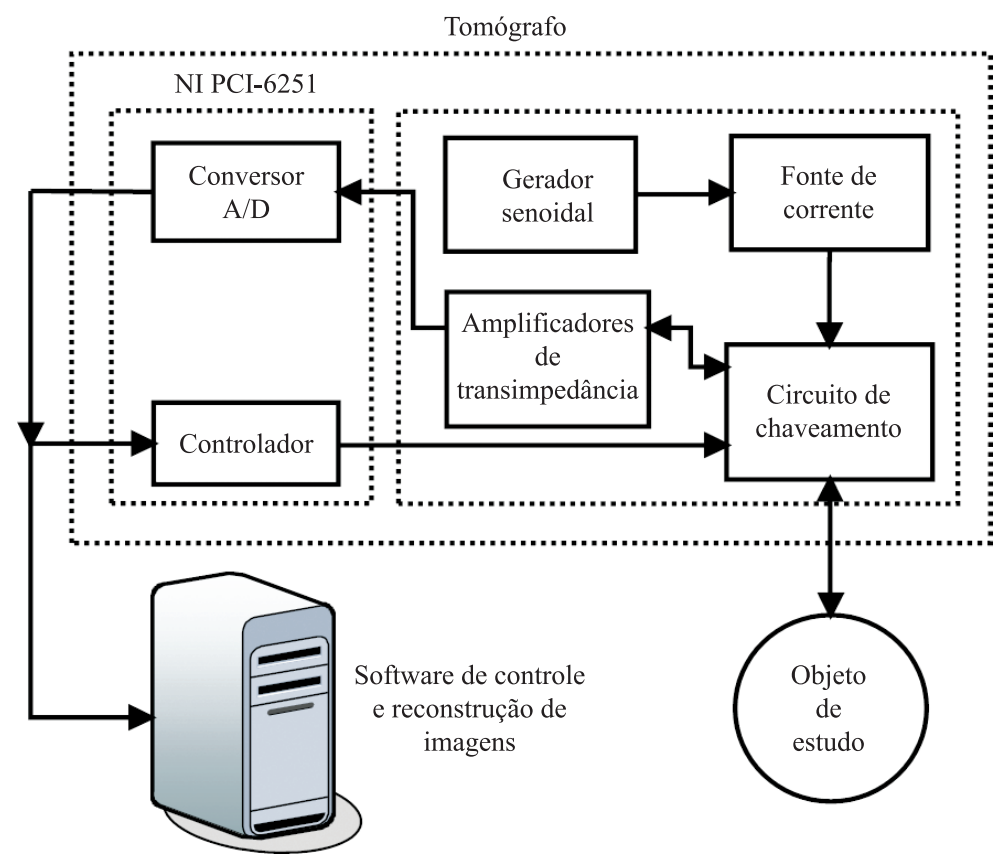

Figura 6. Diagrama de blocos do sistema de EIT em configuração multiterminais utilizado neste trabalho. Figure 6. Block diagram of the multiterminal-based EIT system used in this work.

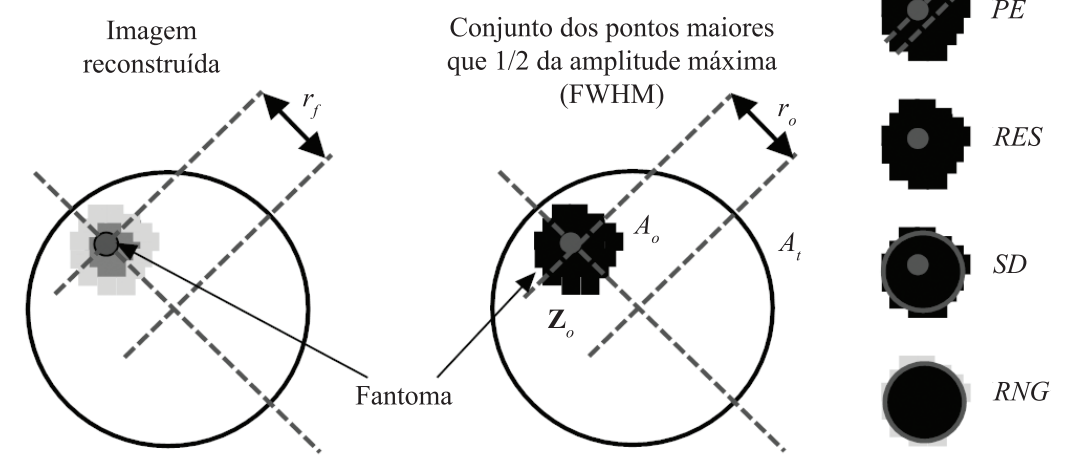

Figura 7. Figuras de mérito para avaliação de imagens de EIT (Adler et al., 2009). A partir da imagem reconstruída é formado o subconjunto $\mathbf{Z}_{0}$ selecionando-se os pixels cuja amplitude ultrapassa $1 / 2$ da amplitude máxima.

Figure 7. Figures of merit to evaluate the EIT images (Adler et al., 2009). The subset $\mathbf{Z}_{0}$ is formed by selecting the pixels whose amplitude is greater than $1 / 2$ of the maximum amplitude. 
meio, a oscilação terá sinal negativo. Altos valores de overshoot ao redor do objeto reconstruído podem induzir a interpretações incorretas da distribuição de condutividade.

Resolução (RES - Resolution): Relaciona o tamanho do objeto reconstruído como uma fração da área total da imagem reconstruída.

$$
R E S=\sqrt{\frac{A_{o}}{A_{t}}}
$$

onde $A_{o}$ corresponde à área do conjunto $\mathbf{Z}_{o}$ e $A_{t}$ à área total da imagem, ambas sendo um somatório dos pixels pertencentes a cada área. Cabe ressaltar que o que se pretende avaliar através dessa figura de mérito é a uniformidade da resolução ao longo do meio e não se o método de reconstrução apresenta altos valores para $R E S$.

\section{Deformação (SD - Shape Deformation):} Quantifica o subconjunto de pixels de $\mathbf{Z}_{o}$ que não está contido no círculo $C$ centralizado no centro de gravidade de $\mathbf{Z}_{0}$ e raio definido tal que a área desse círculo seja equivalente à área $A_{o}$.

$$
S D=\frac{\sum_{k \notin C}\left[\mathbf{Z}_{o}\right]_{k}}{\sum_{k}\left[\mathbf{Z}_{o}\right]_{k}}
$$

onde $\sum_{k}\left[\mathbf{Z}_{o}\right]_{k}$ é justamente a área total $A_{o}$ do objeto reconstruído. Altos valores de $S D$ levam a interpretações equivocadas das imagens.

\section{Resultados}

O algoritmo foi implementado utilizando Matlab ${ }^{\circledR} \mathrm{e}$ as imagens usadas para sua avaliação foram geradas a partir de dados coletados em experimentos relacionados ao deslocamento do fantoma de PVC previamente descrito ao longo de 26 posições igualmente espaçadas sobre um caminho radial. Quatro dessas situações são mostradas na Figura 8, enquanto que o comportamento das figuras de mérito para as 26 posições são representadas na Figura 9.

Adicionalmente, para observar a robustez do algoritmo para mais de um fantoma, foram realizados experimentos com dois e três fantomas, dos quais resultaram as imagens da Figura 10.

\section{Discussão}

Como observado na Figura 9a, o erro de posição oscila, mas não sai da faixa de $\pm 10 \%$ do raio máximo do tanque. Note-se também que à medida que o fantoma se aproxima da borda do tanque o erro de posição tende a diminuir e estabilizar em 5\%, diferentemente do que ocorre nos algoritmos de retroprojeção de Sheffield e no de Gauss-Newton, cujos valores de $P E$ divergem na borda (Adler et al., 2009). Pela Figura 8, observa-se

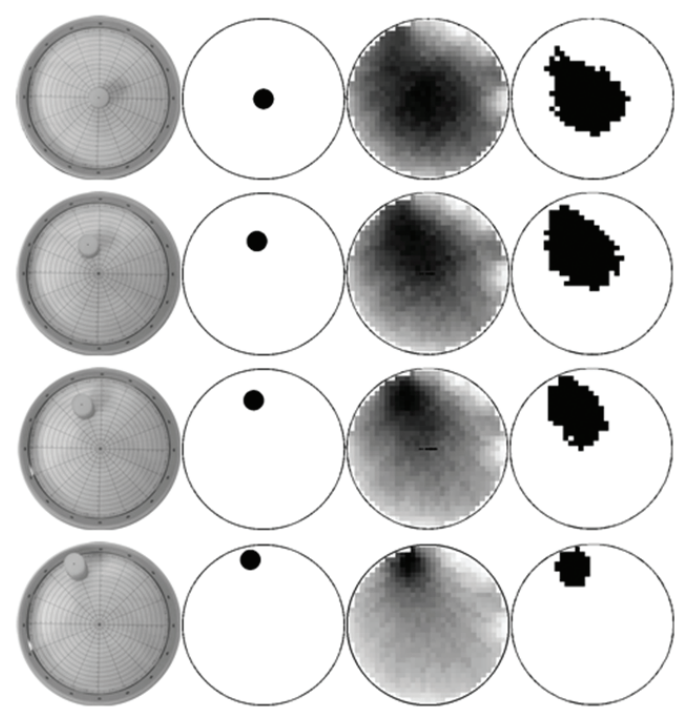

Figura 8. Imagens reconstruídas com o algoritmo para EIT multiterminais. Da esquerda para a direita: imagem real, imagem ideal, imagem reconstruída, imagem segmentada segundo critério FWHM. De cima para baixo: no centro, a $41 \%, 62 \%$ e $83 \%$ do raio em relação ao centro. As imagens correspondem aos dados brutos, sem o uso de filtros.

Figure 8. Reconstructed images using the algorithm developed for multiterminal EIT system. From left to right: real image, ideal image, reconstructed image, segmented image FWHM. From top to bottom: in the center, $41 \%, 62 \%$ and $83 \%$ of the radius from the center. Images correspond to the raw data, without the use of filters.

que quando o fantoma está localizado no centro do tanque há a presença de muitos artefatos de forma desbalanceada em relação ao centro. Como o erro de posição utiliza o centro de gravidade da imagem segmentada, qualquer deformação assimétrica na imagem provoca o aumento de $P E$.

Apesar da resolução apresentar um valor elevado no centro e decrescer na borda, como mostrado no gráfico da Figura 9b, deve-se atentar para o fato de que o que deve ser avaliado nessa figura de mérito é a uniformidade e não o valor de RES. Essa uniformidade não foi observada nos experimentos realizados, apesar do comportamento ser similar ao dos algoritmos de Gauss-Newton e de Graz (Adler et al., 2009).

Quanto à deformação, dado o comportamento das imagens reconstruídas (Figura 8), era de se esperar que ela diminuísse próximo da borda. Apesar da não uniformidade da deformação, nota-se pelo gráfico da Figura 9c que o valor de $S D$ oscila em torno de um valor médio de aproximadamente $12 \%$ e cai bem abaixo de $10 \%$ na borda. Esse comportamento difere bastante do algoritmo de retroprojeção de Sheffield (Adler et al., 2009), que apresenta aumento considerável de $S D$ na borda.

Por fim, embora os valores de $R N G$ (Figura 9d) não sejam baixos no centro, a curva é aproximadamente 

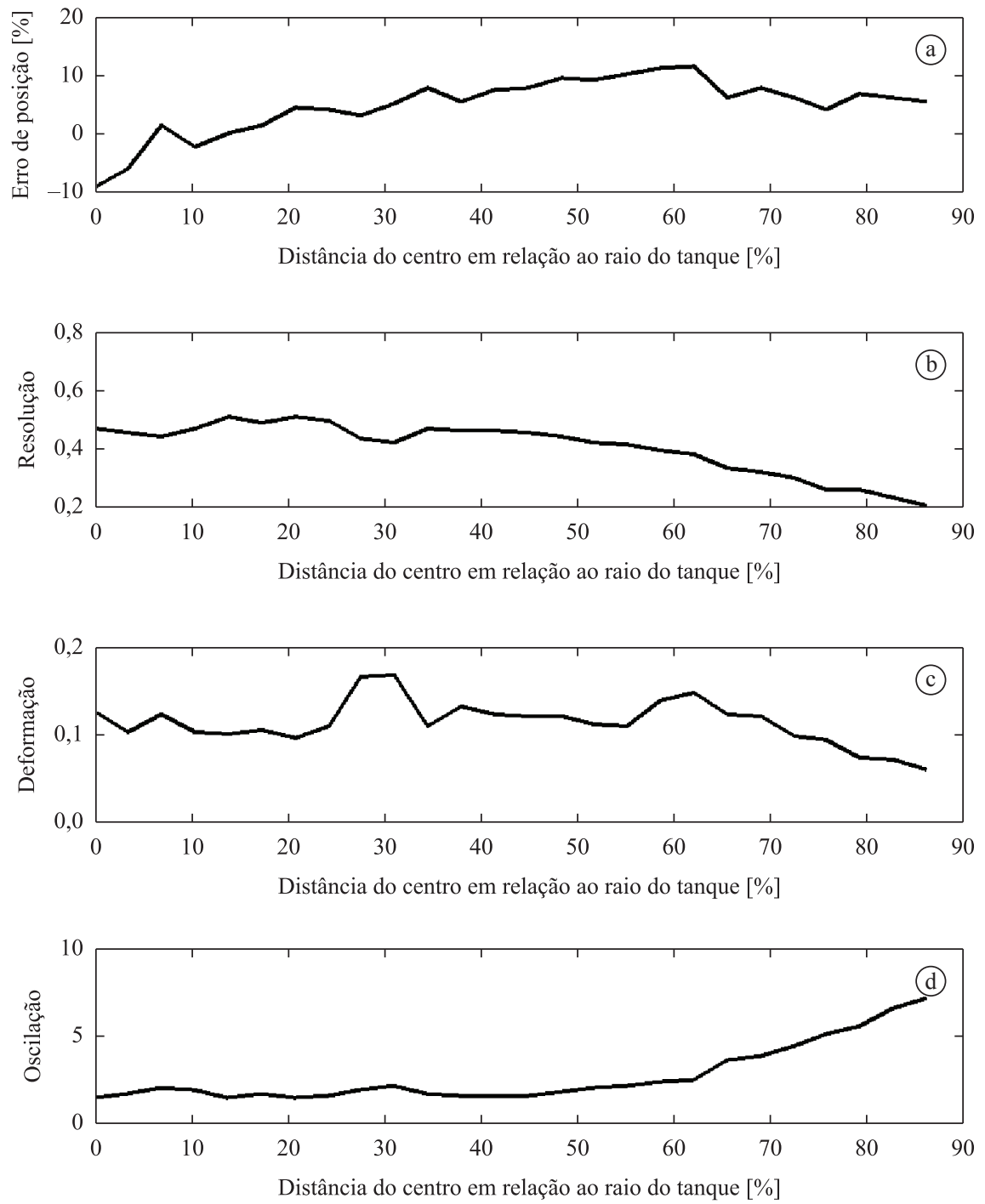

Figura 9. Avaliação do algoritmo segundo as figuras de mérito (a) erro de posição; (b) resolução; (c) deformação; e (d) oscilação. Figure 9. Evaluation of the algorithm according the figures of merit (a) position error; (b) resolution; (c) shape deformation; and (d) ringing.

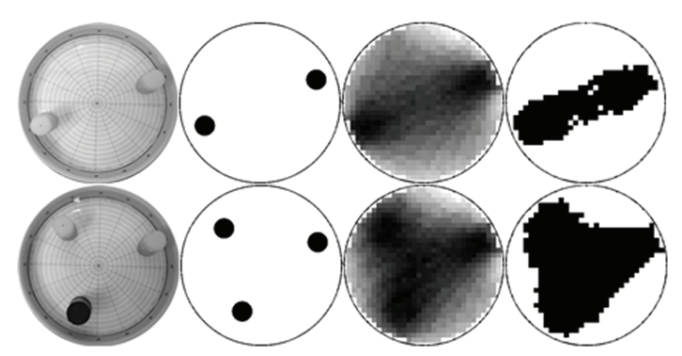

Figura 10. Imagens reconstruídas para dois (acima) e três (abaixo) fantomas. Da esquerda para a direita: imagem real, imagem ideal, imagem reconstruída, imagem segmentada segundo critério FWHM. Figure 10. Reconstructed images for two (above) and three (below) phantoms. From left to right: real image, ideal image, reconstructed image and segmented image FWHM. uniforme até uma distância de $60 \%$ do raio a partir do centro, e a partir daí cresce consideravelmente, como ocorre no algoritmo de retroprojeção de Sheffield (Adler et al., 2009).

Conforme observado na Figura 10, as reconstruções com mais de um fantoma apresentaram baixa nitidez, o que consequentemente interefere na segmentação. Entretanto, deve-se considerar que não foi utilizada nenhuma técnica de filtragem no algoritmo, e que ainda o processo e os critérios de segmentação podem ser aprimorados.

Um novo algoritmo para a reconstrução de imagens de EIT em sistemas multiterminais foi apresentado. 
Este algoritmo é baseado em princípios de analogia eletrostática, não utiliza filtragem na reconstrução e apresenta imagens de EIT comparáveis a outros algoritmos já apresentados na literatura. Uma vez que seu framework de desenvolvimento pode ser considerado simples, seu tempo de construção é otimizado. No entanto, os testes realizados mostraram que o algoritmo apresentado neste trabalho é susceptível a ruídos e maus contatos nos eletrodos, à variação na geometria do tanque e ao descasamento das impedâncias de entrada dos pré-amplificadores de transimpedância. Apesar disso, considerando que esta é uma abordagem alternativa para sistemas multiterminais, conclui-se que os resultados alcançados foram satisfatórios e que são promissores estudos mais aprofundados no sentido de se aperfeiçoar o algoritmo.

\section{Agradecimentos}

Os autores agradecem ao Conselho Nacional de Desenvolvimento Científico e Tecnológico (CNPq) e à Fundação de Amparo à Pesquisa do Estado do Rio de Janeiro (FAPERJ) pelos recursos concedidos para o desenvolvimento deste trabalho, ao aluno Filipe Maia pela importante participação na implementação do hardware, e aos colegas do Departamento de Engenharia de Manutenção da Fiocruz pelo auxílio na confecção dos protótipos utilizados nos experimentos.

\section{Referências}

Adler A, Arnold JH, Bayford R, Borsic A, Brown B, Dixon P, Faes TJC, Frerichs I, Gagnon H, Gärber Y, Grychtol B, Hahn G, Lionheart WRB, Malik A, Patterson RP, Stocks J, Tizzard A, Weiler N, Wolf GK. GREIT: a unified approach to 2D linear EIT reconstruction of lung images. Physiological Measurement. 2009; 30:S35-55. http://dx.doi. org/10.1088/0967-3334/30/6/S0

Ardrey DB, Murphy EK, Isaacson D, Saulnier GJ, Newell JC. Electrical impedance tomography using the finite element method in the mammography geometry. In: Bioengineering Conference (NEBEC) 2011: Proceedings of the IEEE 37th Annual Northeast; 2011 Apr 1-3; Troy, New York. New York; 2011.

Bagshaw AP, Liston AD, Bayford RH, Tizzard A, Gibson AP, Tidswell AT, Sparkes MK, Dehghani H, Binnie CD, Holder DS. Electrical impedance tomography of human brain function using reconstruction algorithms based on the finite element method. NeuroImage. 2003; 20:752-64. http://dx.doi.org/10.1016/S1053-8119(03)00301-X

Barber DC, Brown BH. Applied potential tomography. Journal of Physics E: Scientific Instruments. 1984; 17:723-33. http://dx.doi.org/10.1088/0022-3735/17/9/002

Bertemes-Filho, P. Cálculo do campo elétrico em materiais biológicos usando a técnica das fontes imaginárias. Revista Brasileira de Engenharia Biomédica. 2006; 22(3):227-37.
Borsic A, Halter R, Wan Y, Hartov A, Paulsen KD. Electrical impedance tomography reconstruction for three-dimensional imaging of the prostate. Physiological Measurement. 2010; 31:S1-16. PMid:20647619 PMCid:2994271. http://dx.doi.org/10.1088/0967-3334/31/8/ S01

Brown BH, Barber DC, Seagar AD. Applied potential tomography: possible clinical applications. Clinical Physic and Physiological Measurement. 1985; 6(2):109-21. http:// dx.doi.org/10.1088/0143-0815/6/2/002

Cheney M, Isaacson D, Newell JC, Simske S, Goble J. NOSER: An algorithm for solving the inverse conductivity problem. International Journal of Imaging Systems and Technology. 1990; 2(2):66-75. http://dx.doi.org/10.1002/ ima. 1850020203

Choi MH, Kao TJ, Isaacson D, Saulnier GJ, Newell JC. An algorithm for applying multiple currents using voltage sources in electrical impedance tomography. International Journal of Control, Automation and Systems. 2008; 6(4):613-9.

Durand E. Électrostatique. Saint-Germain: Masson; 1964.

Eyüboglu BM. Electrical impedance imaging, injected current. In: Akay M, editor. Encyclopedia of Biomedical Engineering. New Jersey: Wiley and Sons, Inc.; 2006. p. 1995-205. http:// dx.doi.org/10.1002/9780471740360.ebs0400

Gabriel S, Lau RW, Gabriel C. The dielectric properEITs of biological tissues: II. Measurements in the frequency range $10 \mathrm{~Hz}$ to $20 \mathrm{GHz}$. Physics in Medicine and Biology. 1996; 41:2251-69. PMid:8938025. http://dx.doi. org/10.1088/0031-9155/41/11/002

Halter RJ, Hartov A, Paulsen KD. A broadband highfrequency electrical impedance tomography system for breast imaging. IEEE Transactions on Biomedical Engineering. 2008; 55(2):650-9. PMid:18270001. http:// dx.doi.org/10.1109/TBME.2007.903516

Holder DS. Electrical Impedance Tomography: Methods, history and applications. London: Taylor \& Francis; 2004. http://dx.doi.org/10.1201/9781420034462

Hua P, Woo EJ, Webster JG, Tompkins WJ. Iterative reconstruction methods using regularization and optimal current patterns in electrical impedance tomography. IEEE Transactions on Medical Imaging. 1991; 10(4):621-8. PMid:18222869. http://dx.doi.org/10.1109/42.108598

Isaacson D, Edic P. An algorithm for impedance imaging. In: Annual International Conference of the IEEE Engineering in Medicine and Biology Society: Proceedings of the 14th Annual International Conference of the IEEE Engineering in Medicine and Biology Society; 1992 Oct 29-Nov 1; Paris, France. Paris; 1992. p. 1693. http://dx.doi.org/10.1109/ IEMBS.1992.590089

Kauati AT, Souza MN, Nadal J. A new analytical method for the inverse problem in electrical impedance tomography. In: Annual International Conference of the IEEE Engineering in Medicine and Biology Society: Proceedings of the 20th Annual International Conference of the IEEE Engineering in Medicine and Biology Society; 1998 Oct 29-Nov 1; Hong Kong. Hong Kong; 1998. p. 1044-7. 
Kauati AT, Souza MN. Proceedings of the First Joint BMES/ EMBS Conference; 1999 Oct 13-16; Atlanta, Georgia. Atlanta; 1999. p. 1128.

Linderholm P, Marescot L, Loke MH, Renaud P. Cell culture imaging using microimpedance tomography. IEEE Transactions on Biomedical Engineering. 2008; 55(1): 138-46. PMid:18232355. http://dx.doi.org/10.1109/ TBME.2007.910649

Liston AD. Models and image reconstruction in electrical impedance tomography of human brain function $[\mathrm{PhD}$ thesis]. London: Middlesex University; 2003.

Liu N. ACT4: A high-precision, multi-frequency electrical impedance tomography [PhD thesis]. New York: Faculty of Rensselaer Polytechnic Institute; 2007.

Nebuya S, Mills GH, Milnes P, Brown BH. Indirect measurement of lung density and air volume from electrical impedance tomography (EIT) data. Physiological Measurement. 2011; 32:1953-67. http://dx.doi. org/10.1088/0967-3334/32/12/006

Oh TI, Woo EJ, Holder D. Multi-frequency EIT system with radially symmetric architecture: KHU Mark1. Physiological Measurement. 2007; 28:S183-196. http:// dx.doi.org/10.1088/0967-3334/28/7/S14
Oh TI, Koo H, Lee KH, Kim SM, Lee J, Kim SW, Seo JK, Woo EJ. Validation of a multi-frequency electrical impedance tomography (mfEIT) system KHU Mark1: impedance spectroscopy and time-difference imaging. Physiological Measurement. 2008; 29:295-307. http:// dx.doi.org/10.1088/0967-3334/29/3/002

Ortega JM. Iterative solution of nonlinear equations in several variables. New York: Academic Press; 1970.

Saulnier GJ, Liu N, Tamma C, Xia H, Jao TJ, Newell JC, Isaacson D. An electrical spectroscopy system for breast cancer detection. In: Annual International Conference of the IEEE Engineering in Medicine and Biology Society: Proceedings of the 29th Annual International Conference of the IEEE EMBS; 2007 Aug 23-26; Cité Internationale, Lyon. Lyon; 2007. p. 4154-7. PMid:18002917.

Wilson AJ, Milnes P, Waterworth AR, Smallwood RH, Brown BH. Mk3.5: a modular, multi-frequency successor to the Mk3a EIS/EIT system. Physiological Measurement. 2001; 22:49-54. PMid:11236889. http:// dx.doi.org/10.1088/0967-3334/22/1/307

Yerworth RJ, Bayford RH, Brown B, Milnes P, Conway M, Holder DS. Electrical impedance spectroscopy (EITS) for human head imaging. Physiological Measurement. 2003; 24:477-89. PMid:12812431. http:// dx.doi.org/10.1088/0967-3334/24/2/358

\section{Autores}

Felipe Dalvi-Garcia*, Marcio Nogueira de Souza, Alexandre Visintainer Pino

Programa de Engenharia Biomédica, Instituto Alberto Luiz Coimbra de Pós-Graduação e Pesquisa de Engenharia COPPE, Universidade Federal do Rio de Janeiro - UFRJ, Av. Horácio Macedo, 2030, Prédio do Centro de Tecnologia, Bloco H, Sala 327, Cidade Universitária, CEP 21941-914, Rio de Janeiro, RJ, Brasil. 